MYU Tokyo

S \& M 1176

\title{
Parametric Design and Experimental Research for An Iron-Gallium Alloy Force Sensor
}

\author{
Bo Li, Peng-hui Li, Liang Shu, ${ }^{*}$ and Ding-fang Chen \\ Institute of Intelligent Manufacturing and Control, Wuhan University of Technology, China \\ ${ }^{1}$ The Key Laboratory of Low-Voltage Apparatus Intellectual Technology of Zhejiang, Wenzhou University, China
}

(Received Sepetmber 2, 2015; accepted October 19, 2015)

Keywords: magnetostrictive sensing, optimization, magnetization model, force calibration, susceptibility

A magnetostrictive force sensor that can be used to measure alternating load is presented. Iron-gallium alloy (Galfenol) is used as the sensing element. The sensor was developed based on a closed structure. A finite element method was employed to optimally design the size of the excitation coil and the flux path. Simulation results show that the flux density distribution can be clearly improved after optimization. Energy efficiency analysis shows that the energy efficiency of the proposed sensor can be increased more than 5 times that of the open structure sensor. To study the sensing behavior, a discrete energy-averaged model was developed to analyze the susceptibility change of Galfenol when it was loaded with alternating stress. Experimental results show that the model can predict the change in susceptibility with good accuracy. The linear part of susceptibility was selected to calibrate the sensor with an alternating load of $\pm 1270 \mathrm{~N}$ magnitude. Experiments were conducted to verify the calibration.

\section{Introduction}

Smart materials have properties that change when the materials are exposed to external fields and stress. These special features can be taken advantage of for force-sensing applications, such as piezoelectric sensors ${ }^{(1-3)}$ and magnetostrictive sensors. ${ }^{(4-7)}$ Force sensor designs based on piezoelectric materials were reported in Refs. 1-3. A capacitive pressure sensor was presented in Ref. 1 to measure the chamber pressures of artillery of different calibers. In Ref. 2, a three-axis piezoresistive acceleration force sensor was developed. The piezoresistors were used in resistorcapacitor (RC) oscillators whose oscillation frequencies varied owing to the change in resistance due to an external acceleration force. A piezoelectrical tweezer-style end effector was discussed in Ref. 3. A hysteretic piezoelectric model was used to estimate force for the purpose of self-sensing. Experimental validation showed an average of $12 \%$ error between the self-sensed and the true values. However, piezoelectric materials are too brittle and cannot withstand large tensile loads. ${ }^{(8,9)}$ Also, the materials can only be subjected to one-dimensional compressive forces.

Magnetostrictive materials undergo magnetization changes when exposed to external stress. ${ }^{(10)}$ Giant magnetostrictive materials (Terfenol-D) and iron-gallium alloy (Galfenol) are the two typical magnetostrictive materials that have been studied for force-sensing applications. The inverse magnetostrictive effect was investigated in Refs. 11 and 12. The force-sensing applications

${ }^{\bar{*}}$ Corresponding author: e-mail: shuliangalbert@163.com 
of Terfenol-D were studied in Refs. 5-7. In Ref. 5, a hysteretic nonlinear model of Terfenol-D was developed to describe the inverse magnetostrictive effect. The nonlinear dynamic characteristics of the sensor were studied and experimental results were discussed. In Ref. 6, a Terfenol-D-based sensor was presented for static force measurement. A Hall sensor was integrated into the sensor to measure the flux density change under different static forces. Experimental results showed that the sensor and the proposed model were good at reflecting the static force. Another Terfenol-D-based force sensor was reported in Ref. 7. An open structure was employed to design the flux path, and one-directional compressive stress was applied to the structure to analyze the permeability change of the material. However, the sensor was designed based on an open structure, and a large flux leakage could result in substantial energy waste. Moreover, the brittleness of Terfenol-D limits the application of force sensing. The material can only be subjected to one-dimensional compressive loads. Large tension could cause the failure of materials.

Iron-gallium alloy can be safely loaded with normal or shear stress (up to $500 \mathrm{MPa}$ in tension), and it can be operated as a sensor in the load path. ${ }^{(13)}$ Moreover, this material can be machined, welded, extruded, and deposited into complex geometries. ${ }^{(14-16)}$ These special properties enable Galfenol to be used for measuring continuous alternating loads. The stress-dependent susceptibility of Galfenol under different compressive stresses was investigated in Ref. 7. An analytic model was formulated to study susceptibility in the linear region and experiments were conducted to validate the model. In Ref. 17, magnetization varying with different compressive stresses and stress frequencies was studied. The susceptibilities of Galfenol with different Ga contents were compared to obtain an ideal Ga content for force sensors. In Refs. 18 and 19, the magnetization and strain of the Galfenol alloy were measured under applied stress at constant field. Sensitivity under different applied stresses was discussed. In these studies, the sensing structure was designed based on an open structure. To increase energy efficiency, the flux path design needs to be optimized. Moreover, to measure the alternating loads, the change in susceptibility under different stress levels needs to be studied, and the calibration from susceptibility to external force needs to be investigated experimentally. In this paper, a Galfenol-based force sensor was developed based on a closed structure design. A finite element method was employed to optimally design the size of the excitation coil and the flux path. To study the sensing behavior, a discrete energy-averaged model was developed to analyze the susceptibility change of Galfenol when it was loaded with alternating stress, and experimental calibration was conducted to measure the alternating load with a magnitude of $\pm 1270 \mathrm{~N}$. This paper is organized as follows: structural optimization is discussed in $\S 2$ and the modeling of susceptibility is developed in $\S 3$. Calibration and experimental results are discussed in $\S$ 4 and conclusions are offered in $\S 5$.

\section{Sensor Design}

\subsection{Structural design and parameter study}

The frame of the Galfenol-based force sensor is illustrated in Fig. 1. The sensor consists of different components, including Galfenol rods, shell, base, output shaft, and excitation coil. To apply alternating stress, screw threads are fabricated at the two ends of the Galfenol rod. One end of Galfenol rod is fixed to the base and the other end is connected to the shaft using iron nuts. A biased field is generated with the electromagnetic coil. A strain gauge is mounted on the rod to measure the magnetoelastic property of the material and a pick-up coil is used to measure the magnetization change of the Galfenol. A Hall chip is used to measure the biased field. 


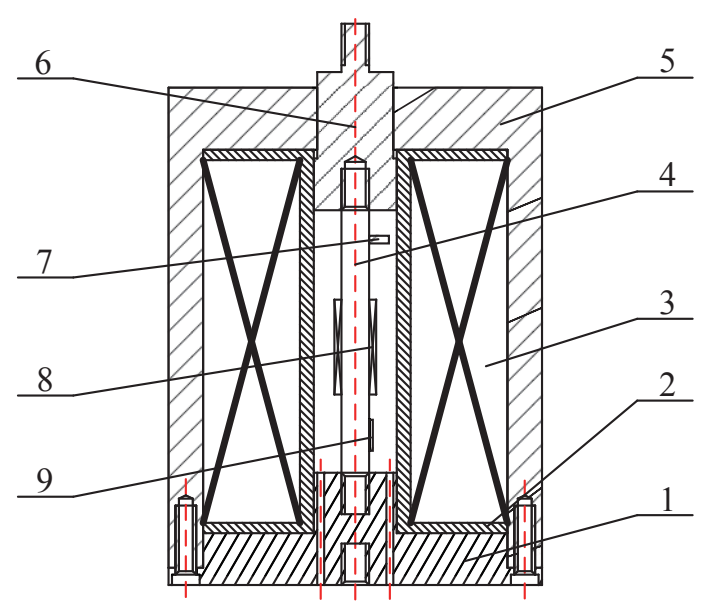

Fig. 1. (Color online) Structure of force sensor. 1. base; 2. coil frameworks; 3. excitation coil; 4. Galfenol rod; 5. shell; 6. output shaft; 7. hall probe; 8. pick-up coil; 9. resistance strain gauges.

It has been discussed that to increase the sensing performance, the electromagnetic component of the sensor needs to be optimized. A finite element method was adopted to analyze the field distribution using different design parameters. In the parametric design, the dimensions that stay constant are shown in Table 1.

In order to implement the parametric design of the electromagnetic component, the following notations are defined:

$$
\alpha_{\mathrm{L}}=\frac{L_{\mathrm{c}}}{L_{\mathrm{g}}}, \beta_{\mathrm{W}}=\frac{W_{\mathrm{c}}}{W_{\mathrm{g}}}
$$

where $L$ and $W$ denote the length and width, and the subscripts $c$ and $g$ denote the coil and Galfenol rod, respectively. Firstly, we assume that the width is fixed and the length of the excitation coil ranges from 80 to $120 \mathrm{~mm}$. The rest of the simulation parameters are shown in Table 2. In order to describe the simulation results properly, we set the origin of the coordinate system in Fig. 1 at the bottom of the Galfenol rod. Simulation results are illustrated in Fig. 2, in which $d_{x}$ on the $x$-axis denotes the distance from the origin. The flux density is selected from the center line of the Galfenol rod.

It is seen from Fig. 2 that the maxima hardly change when $\alpha_{\mathrm{L}}$ is less than 1 . Moreover, only the middle part of the Galfenol demonstrates a uniform field distribution. If $\alpha_{\mathrm{L}}$ is greater than 1 , the field distribution of Galfenol can be markedly improved. Moreover, it is observed from Fig. 2 that the maximum of flux density can be increased if we use a longer coil, provided $\alpha_{\mathrm{L}}$ is larger than 1 .

The effect of the width of the excitation coil was studied for different $\beta_{\mathrm{W}}$ and $\alpha_{\mathrm{L}}$ values. Here, we use three different $\alpha_{\mathrm{L}}$ values: 0.9, 1.0, and 1.1. Simulation results are shown in Fig. 3 when the width of the coil was varied from 16 to $28 \mathrm{~mm}$ ( $\beta_{\mathrm{W}}$ ranges from 2 to 3.5$)$.

It is seen from Fig. 3 that the magnetic flux density of Galfenol increased with increasing width of the coil. From Fig. 3(c), we know that for a longer and wider coil, the flux density in Galfenol is larger and more uniform. However, the size of the electromagnetic component cannot be increased infinitely. Considering the balance between the influencing parameters and the limit of the geometry, we chose $\alpha_{\mathrm{L}}=1.1$ and $\beta_{\mathrm{W}}=2.5$ as the optimal parameters to design the force sensor. 
Table 1

Constant dimensions in the parametric design.

\begin{tabular}{lc}
\hline Parameters & Values (mm) \\
\hline Length of Galfenol rod & 100 \\
Diameter of Galfenol rod & 8 \\
Thickness of base & 15 \\
Thickness of shell & 5 \\
Diameter of output shaft & 12 \\
Length of output shaft & 40 \\
Inner diameter of coil & 14 \\
Inner diameter of coil frameworks & 12 \\
\hline
\end{tabular}

Table 2

Simulation parameters.

\begin{tabular}{lc}
\hline Parameters & Values \\
\hline Relative permeability of Galfenol rod & 90 \\
Materials of base, shell, and output shaft & Electric iron \\
Material of coil framework & Nylon \\
Relative permeability of electric iron & 1000 \\
Relative permeability of nylon & 1 \\
Current density in excitation coil & $2.5 \times 10^{5} \mathrm{~A} / \mathrm{m}^{2}$ \\
\hline
\end{tabular}

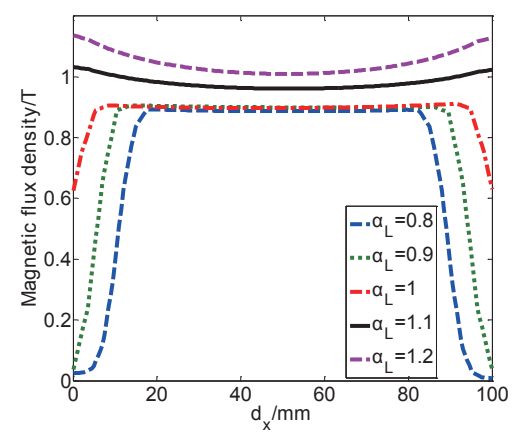

Fig. 2. (Color online) Distribution of magnetic flux density for different $\alpha_{\mathrm{L}}$ values.

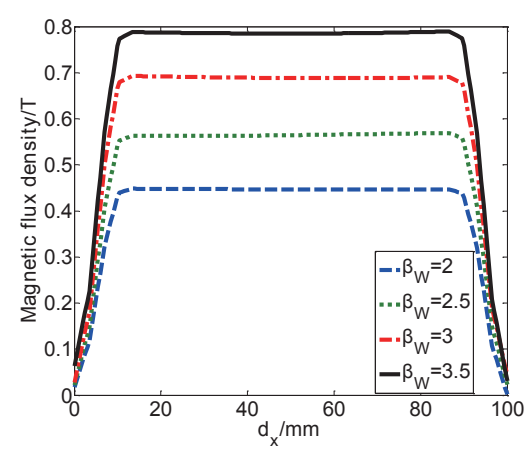

(a)

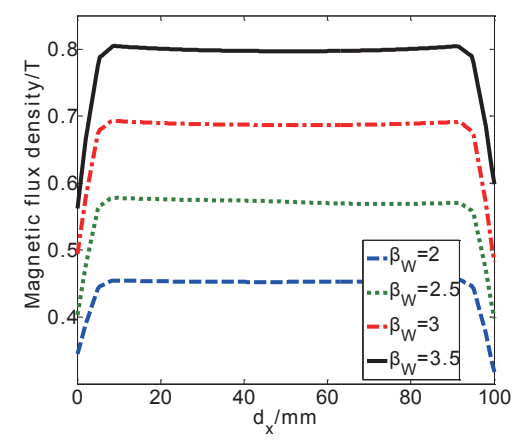

(b)

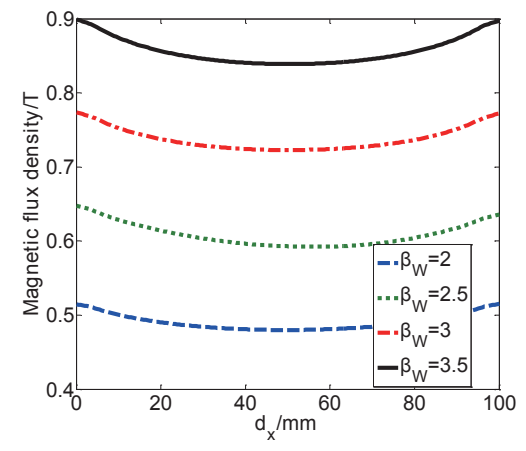

(c)

Fig. 3. (Color online) Distribution of magnetic flux density for different $\alpha_{\mathrm{L}}$ and $\beta_{\mathrm{W}}$ values. (a) $\alpha_{\mathrm{L}}=0.9$, (b) $\alpha_{\mathrm{L}}$ $=1.0$, and (c) $\alpha_{\mathrm{L}}=1.1$. 


\subsection{Comparative simulations}

In this section, we discuss a comparative simulation conducted by comparing the proposed structure with the open structure. The sizes of the Galfenol rods are taken as the same, with the dimensions shown in Table 1. In order to demonstrate the simulation performance of the two structures, the ampere-turns in the simulations were set as the same with a value of $43.7 \mathrm{kA} / \mathrm{m}$. The sensor geometry based on an open structure is illustrated in Fig. 4.

The flux path is composed of the Galfenol rod and silicon steel. The excitation coils are assembled at the sides of the Galfenol. Simulation results are shown in Fig. 5. In the simulation, the length of Galfenol was $100 \mathrm{~mm}$ and its diameter was $8 \mathrm{~mm} ; \beta_{\mathrm{W}}$ was selected as 3 for the open structure. It is seen from Fig. 5 that the flux density in the central part of the Galfenol is much greater than at the two ends.

The flux density along the $x$-axis of the Galfenol rod is plotted in Fig. 6. It is seen from Fig. 6(b) that the flux density distribution of the proposed structure is more uniform than that of the open structure. Moreover, the magnitude of the flux density is larger in Fig. 6(b), even though a wider coil $\left(\beta_{\mathrm{w}}=3\right)$ is used in Fig. 6(a). In Fig. 6(a), it is seen that the magnetization changes direction at the two ends of the Galfenol because the two ends of the rod are not located in the closed loop flux

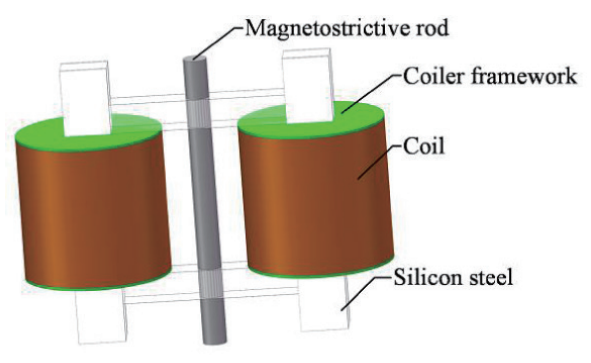

Fig. 4. (Color online) Open structure.

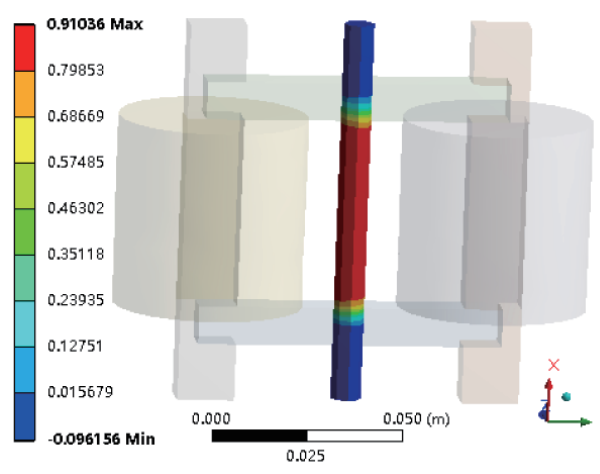

(a)

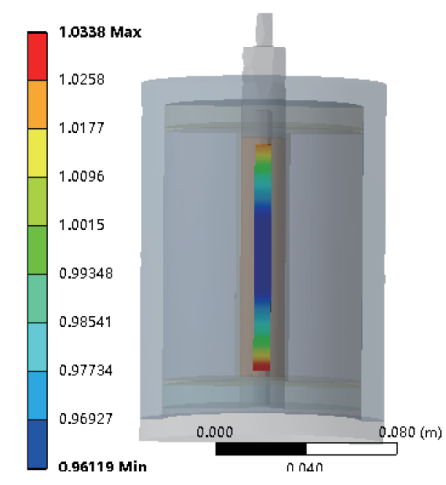

(b)

Fig. 5. (Color online) Flux density distribution of Galfenol. (a) Open structure and (b) proposed structure. 


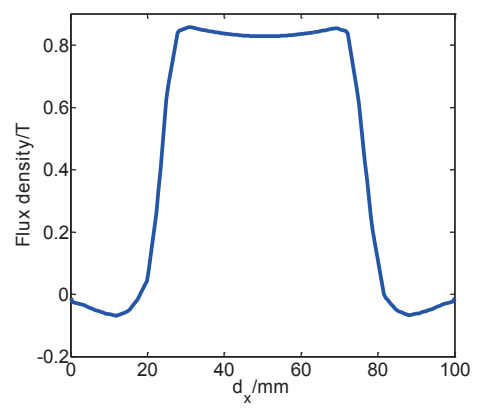

(a)

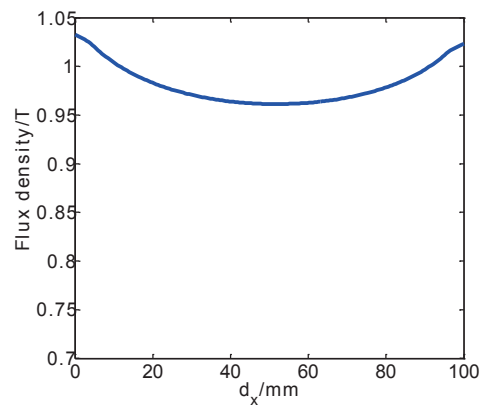

(b)

Fig. 6. (Color online) Flux density along the $x$-axis of Galfenol. (a) Open structure and (b) proposed structure.

path. This directional change of magnetization could affect the sensing performance of Galfenol.

To further study the difference between the two structures, the energy efficiencies of the two structures were calculated according to the following definition,

$$
\eta=\frac{W}{W_{\mathrm{T}}} \times 100 \%
$$

where $W$ denotes the magnetic energy of Galfenol and $W_{\mathrm{T}}$ is the total magnetic energy of the structure. Comparative results are shown in Table 3 for different applied current densities. It is shown in Table 3 that the energy efficiency of the proposed force sensor is approximately 5 times that of the open structure because the air leakage of the proposed structure is low and the energy efficiency can be increased.

\section{Magnetization Model of Galfenol}

To study the sensing behavior, a discrete energy-averaged model ${ }^{(20)}$ was developed to analyze the susceptibility change of Galfenol when it is loaded with alternating stress. The internal energy of the magnetic domain can be expressed as

$$
U(\boldsymbol{m})=K_{4}\left(m_{x}^{2} m_{y}^{2}+m_{y}^{2} m_{z}^{2}+m_{z}^{2} m_{x}^{2}\right)
$$

where $\boldsymbol{m}$ is the directional vector of the internal crystals of the magnetic domain, and $K_{4}$ is the 4thorder anisotropy coefficient. Gibbs free energy is

$$
G(\boldsymbol{H}, \boldsymbol{T})=U(\boldsymbol{m})-\boldsymbol{S} \cdot \boldsymbol{T}-\mu_{0} M_{\mathbf{s}} \boldsymbol{m} \boldsymbol{H},
$$

where $H$ is the vector of the magnetic field, $\boldsymbol{T}$ is the stress tensor, $\boldsymbol{S}$ is the strain, $\mu_{0}$ is the permeability of free space, and $M_{\mathrm{S}}$ is the saturation magnetization. The directional vector can be solved by minimizing the energy [Eq. (4)]. It is assumed that the material is composed of a collection of Stoner-Wohlfarth (SW) particles, ${ }^{(21)}$ and the macroscale magnetization is calculated as the summation of the domain magnetization, weighted by the volume fraction of particles, 
Table 3

Magnetic energies and energy efficiencies of the two structures.

\begin{tabular}{lcccc}
\hline & Applied current density $\left(\mathrm{A} / \mathrm{m}^{2}\right)$ & $1 \times 10^{6}$ & $3 \times 10^{6}$ & $6 \times 10^{6}$ \\
\hline & Magnetic energy density of Galfenol $\left(\mathrm{J} / \mathrm{m}^{3}\right)$ & 702 & 4959 & 19216 \\
Open structure & Magnetic energy of Galfenol $(\mathrm{J})$ & 0.00225 & 0.02016 & 0.08892 \\
& Total magnetic energy of system $(\mathrm{J})$ & 0.01866 & 0.187 & 0.768 \\
& Energy efficiency $(\%)$ & 12.06 & 10.78 & 11.577 \\
\hline \multirow{3}{*}{ Proposed structure } & Magnetic energy density of Galfenol $\left(\mathrm{J} / \mathrm{m}^{3}\right)$ & 7867 & 69784 & 310000 \\
& Magnetic energy of Galfenol $(\mathrm{J})$ & 0.032 & 0.313 & 0.827 \\
& Total magnetic energy of system $(\mathrm{J})$ & 0.049 & 0.481 & 1.241 \\
& Energy efficiency $(\%)$ & 64.2 & 65.1 & 66.6 \\
\hline
\end{tabular}

$$
\boldsymbol{M}=M_{\mathbf{s}} \sum_{k=1}^{\gamma} \xi^{k} \boldsymbol{m}^{k}
$$

where $\gamma$ denotes the possible orientations in the thermodynamic equilibrium and $\xi^{k}$ is the volume fraction of the domain. The volume fraction can be calculated using the following equation,

$$
\xi^{k}=\frac{\exp \left(-G^{k} / \Omega\right)}{\sum_{j=1}^{\gamma} \exp \left(-G^{k} / \Omega\right)},
$$

where $\Omega$ is the Armstrong smooth factor.

Experiments were conducted to verify the model calculations. The prototype of the sensor is illustrated in Fig. 7 and the experimental setup is shown in Fig. 8. A fatigue testing machine was used to supply the continuous alternating load. Magnetic flux density $B$ was measured using a pickup coil midway along the length of the Galfenol rod. The induced voltage was generated according to Faraday's law of induction,

$$
V(t)=-N \frac{d \varphi(t)}{d t}=-N A \frac{d B(t)}{d t}
$$

where $V(t)$ is the induced voltage, $N$ is the nuber of turns of pick-up coil, $\varphi(t)$ is the flux, and $A$ is the cross-section of the coil. If $V(t)$ is measured, we can calculate the flux density by the following integration,

$$
B(t)=-\frac{1}{N A} \int_{0}^{t} V(\tau) d \tau .
$$

We used a Lakeshore 480 flux meter to do the above integration. The magnetic field $H$ was measured at the surface of the rod, midway along the $x$-axis, by a Hall probe. Strain was determined using a resistance strain gauge that was pasted on the surface of the rod. Susceptibility $\chi_{\mathrm{e}}$ was calculated using the following equation, 


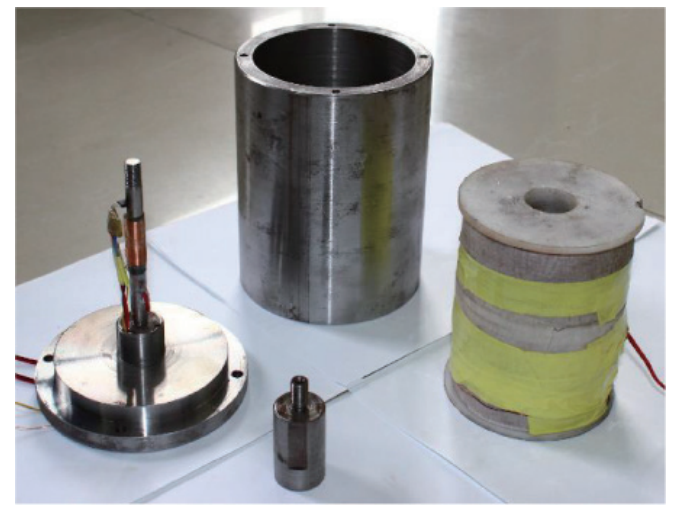

Fig. 7. (Color online) Prototype of the sensor.

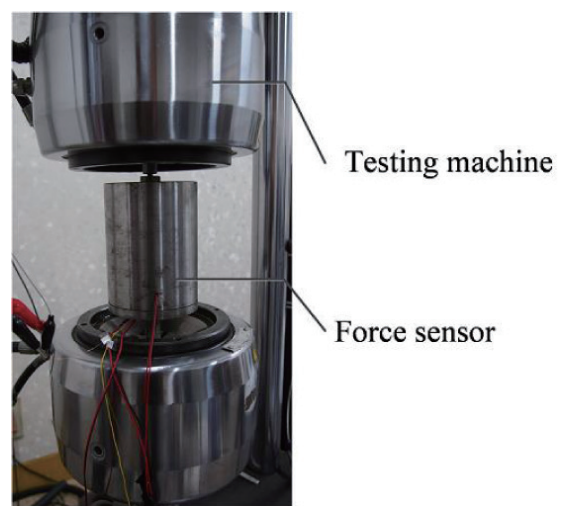

Fig. 8. (Color online) Experimental setup.

$$
\chi_{\mathrm{e}}=\frac{B}{\mu_{0} H}-1,
$$

where $\mu_{0}$ is the permeability of vacuum. $B$ and $H$ are measured values.

To run the simulations, the parameters were selected as follows: $\Omega=1200, \mu_{0} M_{\mathrm{S}}=1.6 \mathrm{~T}, K_{4}$ $=30 \mathrm{~kJ} / \mathrm{m}^{3}$, and $T_{0}=15 \mathrm{MPa}$. Once the material magnetization was determined from Eq. (5), the magnetic susceptibility could be obtained as $\chi_{\mathrm{m}}=M / H$. Here the magnetization direction was selected along the length of the Galfenol rod. A comparison of the results for $\chi_{\mathrm{e}}$ and $\chi_{\mathrm{m}}$ is shown in Fig. 9. It is shown in Fig. 9 that the model prediction has good agreement with the experimental data. The susceptibility of Galfenol demonstrates a nonlinear change according to the change in external force. The change in susceptibility includes the dead zone, the linear zone, and the saturated zone; three zones of susceptibility arise from the hysteresis of Galfenol alloy. The linear zone of susceptibility can be selected to implement the force sensing.

\section{Force Calibration}

It was discussed in $\S 3$ that Galfenol has a stress-dependent susceptibility. However, this susceptibility shows nonlinear behavior according to the external stress. In order to implement the force sensing, we selected the linear zone in Fig. 9 to calibrate the alternating load. In the linear zone, the change in susceptibility according to stress can be described by the following equation, ${ }^{(10)}$

$$
\chi(T)=\frac{\mu_{0} M_{\mathrm{s}}^{2}}{2 K_{4}-3 \lambda_{100} T},
$$

where $\lambda_{100}$ is the magnetostrictive coefficient in the $<100>$ direction. The magnetic susceptibility is measured as discussed in $\S 3$. The sensing force can be calculated as follows, 


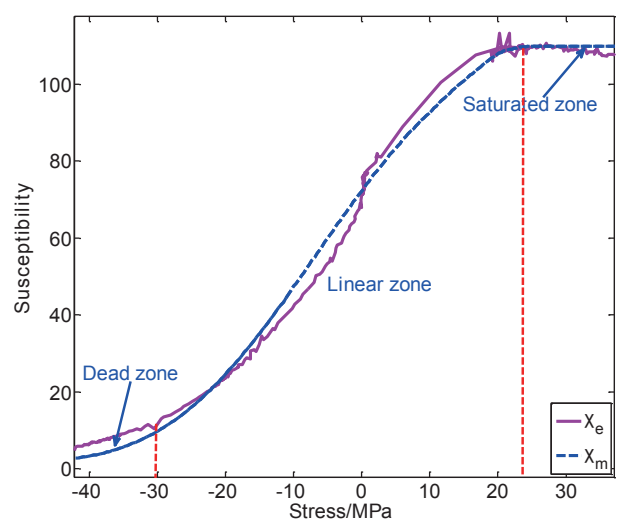

Fig. 9 (Color online) Susceptibility versus stress.

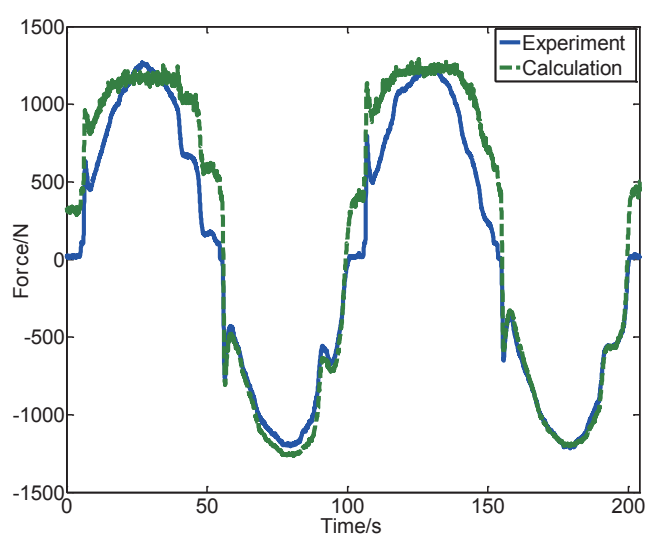

Fig. 11. (Color online) Force measurement.

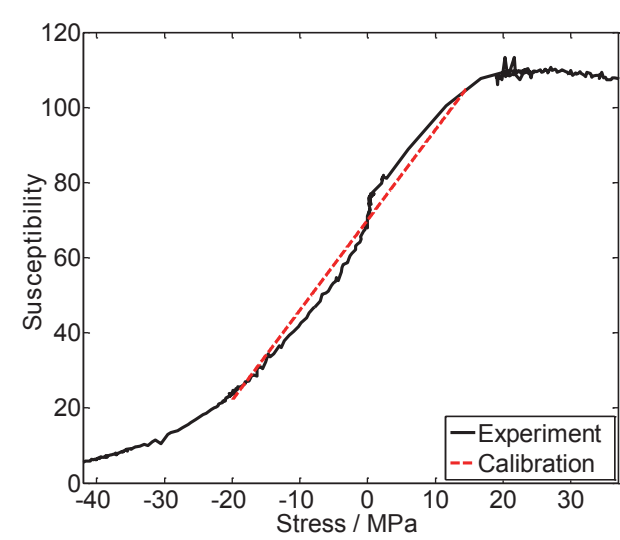

Fig. 10. (Color online) Calibration result of linear susceptibility.

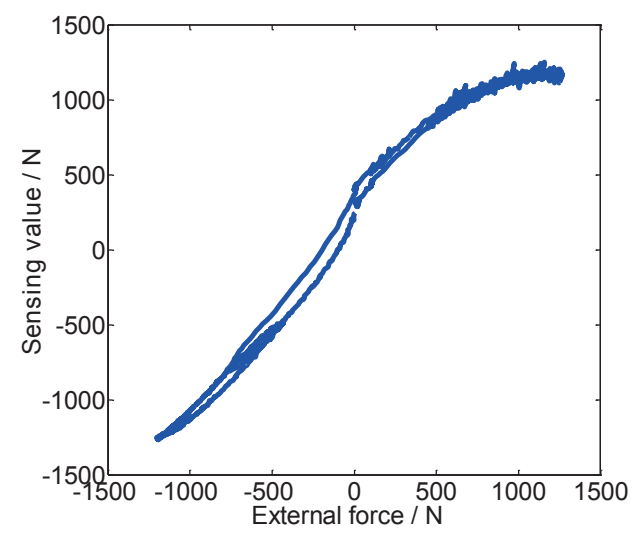

Fig. 12. (Color online) Linearity of the sensor.

$$
F_{\mathrm{s}}=A_{\mathrm{s}} \frac{2 \chi_{\mathrm{e}} K_{4}-\mu_{0} M_{\mathrm{s}}^{2}}{3 \chi_{\mathrm{e}} \lambda_{100}}
$$

where $A_{\mathrm{s}}$ is the cross-sectional area of the Galfenol rod. The linear zone of the curve was chosen to calibrate the force sensing. The linear susceptibility was calculated with Eq. (10), and the comparison with experimental measurement is illustrated in Fig. 10. It is seen that the linear calculation of susceptibility is consistent with the linear region of the experimental measurement. If the susceptibility is measured, we can use Eq. (11) to calibrate the external force.

The calibration result in time domain is illustrated in Fig. 11. The magnitude of applied force is $\pm 1270 \mathrm{~N}$. It can be seen from the figure that the proposed sensor is capable of measuring continuous alternating loads. Since we are using a linear susceptibility equation to calibrate the force, most of the errors come from the hysteretic nonlinearity of the material. It is also seen from Fig. 11 that there are sharp drifts in the external force, which arise from disturbances of the testing machine. It is observed that these random disturbances can still be measured using the proposed sensor. The linearity of the sensor is illustrated in Fig. 12. The $x$-axis is the external force and the $y$-axis is the 
sensing value from the sensor. It can be seen that the sensor demonstrated dapproximately linear behavior. The hysteresis loop in Fig. 12 comes from the instinctive hysteretic property of Galfenol. The linearity is good when the external force is relatively small. The material nonlinearity affect the sensing performance when the force gets larger.

\section{Conclusions}

A Galfenol-based force sensor was proposed for the accurate measurement of alternating load. The sensing structure was optimized by the finite element method. The optimized design parameters were selected as $\alpha_{\mathrm{L}}=1.1$ and $\beta_{\mathrm{W}}=2.5$. Simulation showed that the energy efficiency of the proposed sensor can be increased more than 5 times over that of the open structure sensor. A discrete energy-averaged model was developed to analyze the susceptibility change of Galfenol. Experimental results showed that the model can predict the change in susceptibility with good accuracy. The linear region of the susceptibility was selected to calibrate the sensor with an alternating load of $\pm 1270 \mathrm{~N}$. Experiments showed that the proposed sensor is capable of measuring continuous alternating loads with good accuracy.

\section{Acknowledgements}

This work was supported in part by the National Natural Science Foundation of China under Grant 51205293 and Grant 51175395, in part by the Natural Science Foundation of Zhejiang Province under Grant LY15E050011, and in part by the China Postdoctoral Science Foundation under Grant 2015M571904.

\section{References}

X. E. Li, J. Zu, T. H. Ma, and P. Xu: Sens. Mater. 23 (2011) 277.

Y. Chiu, T. C. Huang, and H. C. Hong: Sens. Mater. 26 (2014) 95.

M. Timonthy and U. Jun: IEEE/ASME Trans. Mechatron. 19 (2014) 755.

4 Y. Miwa, J. Shin, Y. Hayashi, S. Hashi, and K. Ishiyama: IEEE. Trans. Magn. 51 (2015) 1.

5 Z. W. Zhu, W. D. Zhang, and J. Xu: Sens. Mater. 26 (2014) 319.

6 Z. Y. Jia, H. F. Liu, F. J. Wang, W. Liu, and C. Y. Ge: Measurement. 44 (2011) 88.

7 Q. X. Yang, R. G. Yan, C. Z. Fan, H. Y. Chen, F. G. Liu, and S. Liu: IEEE. Trans. Magn. 43 (2007) 1437.

8 D. K. Kleinke and H. M. Uras: Rev. Sci. Instrum. 65 (1994) 1699.

9 B. P. Baillargeon and S. S. Vel: J. Intell. Mater. Syst. Struct. 16 (2005) 517.

10 P. G. Evans and M. J. Dapino: J. Appl. Phys. 108 (2010) 074517.

11 S. Chakraborty and G. R. Tomlinson: Smart. Mater. Struct. 12 (2003) 763.

12 Z. Zhang, J. Q. Mao, and K. M. Zhou: Sci. China Technol. Sci. 56 (2013) 656.

13 L. Shu, L. M. Headings, M. J. Dapino, D. F. Chen, and Q. G. Lu: J. Intell. Mater. Syst. Struct. 25 (2014) 187.

14 J. Atulasimha and A. B. Flatau: Smart. Mater. Struct. 20 (2011) 43.

15 A. E. Clark, W. F. Marilyn, J. B. Restorff, and T. A. Lograsso: Mater. Trans. 43 (2002) 881.

16 R. A. Kellogg: Development and Modeling of Iron-Gallium Alloys (Iowa State University, Iowa, 2003) Chap. 2.

17 L. Weng, B. W. Wang, M. J. Dapino, Y. Sun, L. Wang, and B. Z. Cui: J. Appl. Phys. 113 (2013) 17A917.

18 P. G. Evans and M. J. Dapino: J. Magn. Magn. Mater. 330 (2013) 37.

19 A. Mahadevan, P. G. Evans, and M. J. Dapino: Appl. Phys. Lett. 96 (2010) 012502.

20 P. G. Evans and M. J. Dapino: J. Appl. Phys. 105 (2009) 113901.

21 F. Liorzou, B. Phelps, and D. L. Atherton: IEEE Trans. Magn. 36 (2000) 418. 